\title{
P0323 - Effects of oral glucose tolerance test and steamed bread meal test on serum 1,5-anhydroglucitol levels
}

J. Zhou, H. Su, Y. Wang, J. Yin, X. He, L. Ying, X. Ma, Y. Bao, W. Jia.

Department of Endocrinology and Metabolism, Shanghai Jiao Tong University Affiliated Sixth People's Hospital; Shanghai Clinical Center for Diabetes; Shanghai Key Clinical Center for Metabolic Disease; Shanghai Diabetes Institute; Shanghai Key Laboratory of Diabetes Mellitus, Shanghai, China.

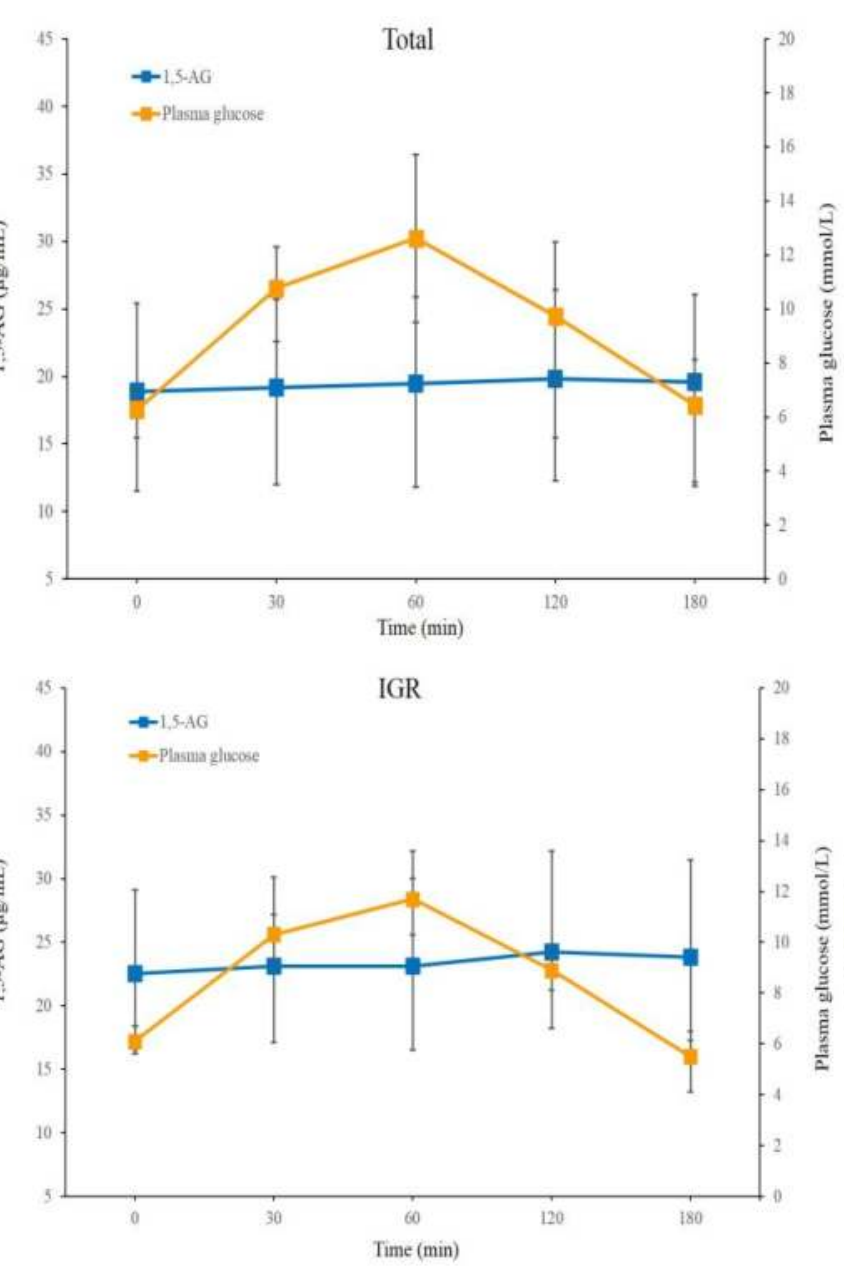

Background

Assessments of biochemical parameters in fasting blood sample have long been the norm. However, "nonfasting" measurements of clinical monitoring indicators have been added to the current research hotpot. Previous studies showed that serum 1,5-anhydroglucitol (1,5-AG) levels were significantly reduced in patients with diabetes mellitus (DM). However, the acute response of 1,5-AG to a glucose load remains unclear, which is important for its non-fasting measurement.

\section{Aim}

This study was conducted to explore acute changes in 1,5-AG levels after a oral glucose tolerance test (OGTT) or a steamed bread meal test (SBMT) and evaluate concordance between fasting and non-fasting levels of 1,5-AG. Simultaneous agreement of 1,5-AG with glycated hemoglobin (HbA1c) and that with glycated albumin (GA) were further compared.

\section{Method}

The study population included Shanghai community residents without a prior history of DM. All of the participants underwent a $75 \mathrm{~g}$ OGTT or $100 \mathrm{~g}$ SBMT. Fasting and postload levels of HbA1c, GA, 1,5-AG, and insulin were measured. Statistical analysis methods included intra-class correlation coefficient, rate of agreement analysis, mountain plot, Passing-Bablok regression, and Bland-Altman difference plots.

\section{Result}

(1) In total of 681 subjects who underwent an OGTT, serum 1,5-AG levels were slightly elevated after a glucose load.
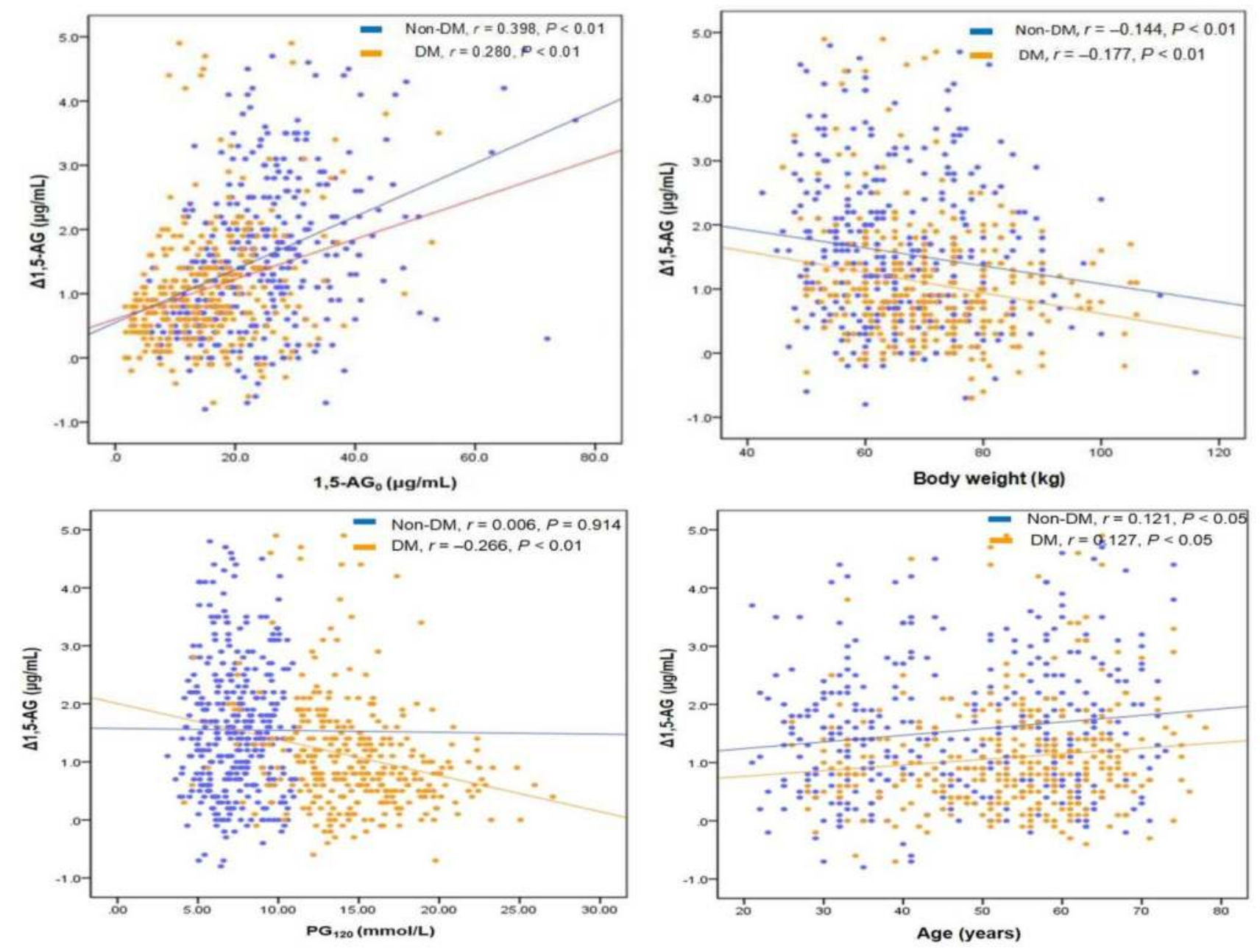

Regression analysis showed that body weight was negatively associated with the range of variation in the postload 1,5-AG level $(\Delta 1,5-A G)$. There is a strong and positive association between baseline $1,5-\mathrm{AG}$ and $\Delta 1,5-$ $\mathrm{AG}$, but not between $\Delta 1,5-\mathrm{AG}$ and insulin levels. The ratio of the $\Delta 1,5-\mathrm{AG}$ to the baseline $1,5-\mathrm{AG}$ was higher in participants with DM (7.3\% [3.4-11.5\%]) than in those without DM (6.2\% [3.6-9.2\%]).

(2) Among 104 Shanghai community participants, the levels of 1,5-AG slightly increased from 30 to $120 \mathrm{~min}$ after SBMT. Consistency analyses of 1,5-AG after SBMT showed good stability. In the same circumstances, however, stability of HbA1c and GA were better than that of 1,5-AG, of which HbA1c is the best.

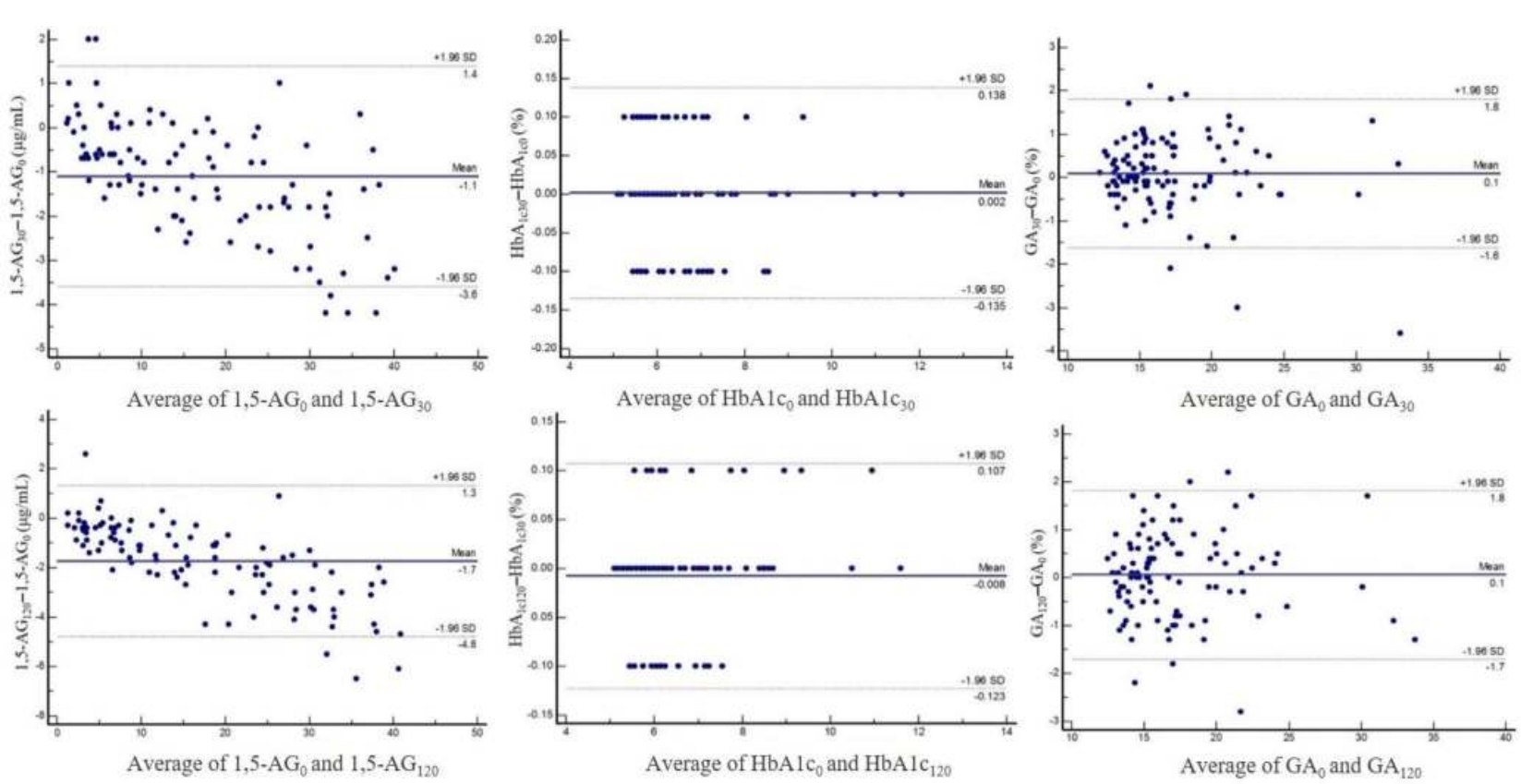

\section{Conclusion}

The present study demonstrated that, unlike the decrease with long-term hyperglycemia, serum 1,5-AG levels were slightly elevated by 6-7\% after a glucose load. Agreement analysis provide a basis for the application of measuring 1,5-AG in non-fasting blood sample to clinical practice. Both $\mathrm{HbA} 1 \mathrm{c}$ and GA stabilities were superior to that of 1,5-AG after SBMT.

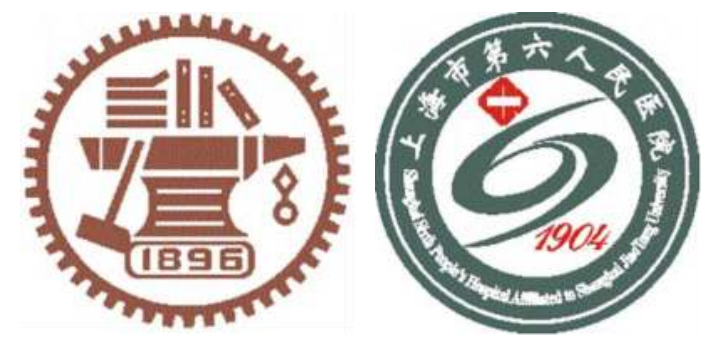

